\title{
Multiple Sclerosis Revealed by Anterior Uveitis
}

\author{
Sidi Dahi, Mehdi Khamaily, Joumany Brahim Salem, Imane Tarib, Mounia Bouchaar, \\ Rachid Zerrouk, Yassine Mouzari, Fouad El Asri, Karim Reda, Abdelbarre Oubaaz
}

\section{ABSTRACT}

Multiple sclerosis is an autoimmune inflammatory disease of the central nervous system, leading to the formation of foci of demyelination. Ocular involvement is quite frequent and multiple, dominated by inflammatory optic neuropathies, oculomotor nerve damage, nystagmus and uveitis. We report an atypical case of multiple sclerosis revealed by an anterior unilateral synechiatic hypertensive uveitis in a young patient of 27 years.

Keywords: Uveitis, muliples sclerosis, demyelination.
Published Online: October 17, 2020

ISSN: $2593-8339$

DOI: $10.24018 /$ ejmed.2020.2.5.508

Sidi Dahi *

Mohammed V Military Hospital

Mohammed V University, Morocco.

(e-mail: dahisidi2014@gmail.com)

Mehdi Khamaily

Mohammed V Military Hospital, Mohammed V University, Morocco.

Joumany Brahim Salem

Mohammed V Military Hospital,

Mohammed V University, Morocco.

Imane Tarib

Mohammed V Military Hospital

Mohammed V University, Morocco.

Mounia Bouchaar

Mohammed V Military Hospital,

Mohammed V University, Morocco.

Rachid Zerrouk

Mohammed V Military Hospital,

Mohammed V University, Morocco.

Yassine Mouzari

Mohammed V Military Hospital,

Mohammed V University, Morocco.

Fouad El Asri

Mohammed V Military Hospital,

Mohammed V University, Morocco.

Karim Reda

Mohammed V Military Hospital,

Mohammed V University, Morocco.

Abdelbarre Oubaaz

Mohammed V Military Hospital,

Mohammed V University, Morocco.

*Corresponding Author

\section{INTRODUCTION}

Multiple sclerosis is an autoimmune inflammatory disease of the central nervous system, leading to the formation of foci of demyelination which manifests as intermittent and recurrent inflammatory episodes, of unpredictable course, and the etiology of which is not yet understood. Ocular involvement is quite frequent and multiple and sometimes inaugural, dominated by inflammatory optic neuropathies, oculomotor damage, nystagmus and uveitis.

\section{CASE REPORT}

We report the case of a young patient of 27 years, who presented to the ophthalmology consultation for a 2nd painful red eye episode. The ophthalmological examination found visual acuity with correction to 12/20 Right Eye, 20/20 Left Eye, intraocular pressure at Goldamn Tonometer: 25 mmhg Right Eye, 16 mmhg Left Eye.

With the slit lamp:

OD: Slight edema of the cornea, fluorescein test negative, retro-descemetics precepitated in the lower part, irido-lens synechia over $360^{\circ}$, without visible iris nodule (Fig. 1).

OG: clear cornea, normal anterior chamber with sequelae of irido-lens synechiae (Fig. 2).

At OF: No signs of intermediate uveitis or retinal vasculitis, or retinal focus with a perfectly normal papilla.

The questioning revealed the notion of disturbed thermal sensitivity of the lower limbs, without other extra ocular signs.

A cerebral-medullary MRI was requested objectifying: Lesions in cerebral-medullary hypersignal (Fig. 3) with an inflammatory cerebrospinal fluid at the lumbar puncture, 
while favor an active form of multiple sclerosis.

After a normal inflammatory and infectious assessment, the patient was put on symptomatic treatment (local corticosteroid therapy, local hypotonics and dilation with atropine) and was referred to neurology for additional management.

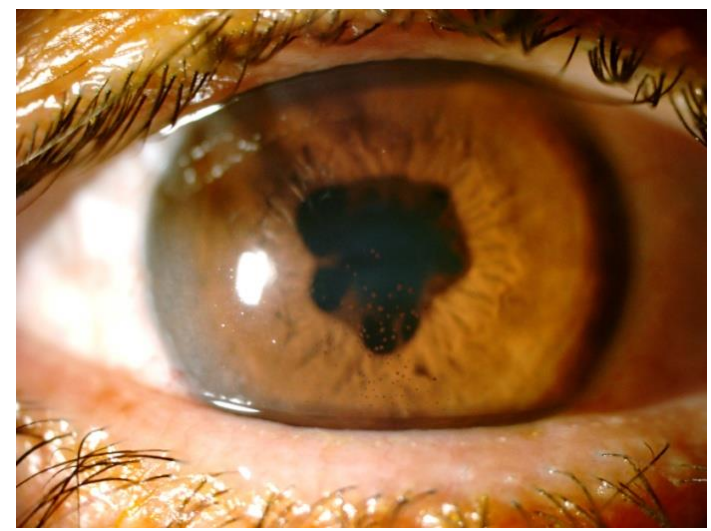

Fig. 1. Right Eye: corneal edema with retro descmetic precipitates and irido-lens synechiae.

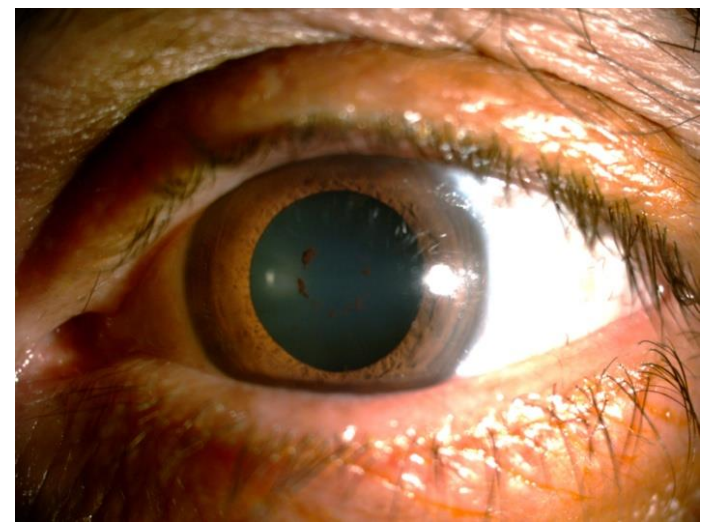

Fig. 2. Left Eye: clear cornea with sequelae of irido-lens synechiae.
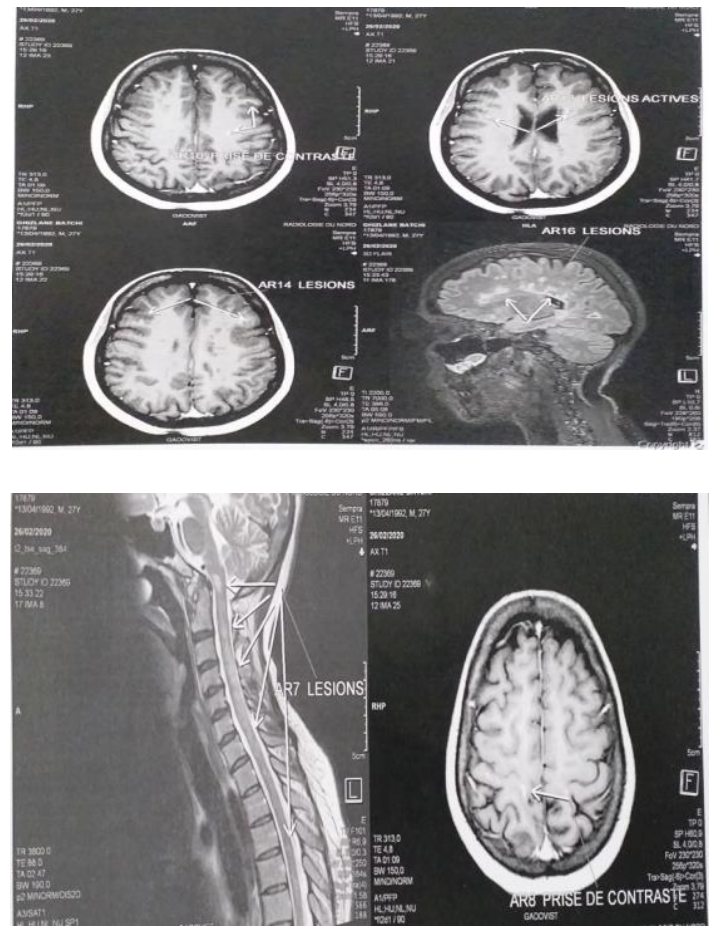

Fig. 3. Magnetic resonance imaging of the brain axial view showing multiple periventricular demyelinating lesions (white arrows).

\section{DISCUSSION}

Multiple sclerosis is the most debilitating neurological disorder affecting young patients in full physical activity between 20 and 40 years [1], with a predominance of women [2] and whose main risk factors are smoking, obesity, low exposure to ultraviolet rays, vitamin D deficiency as well as infections including the Epstein Bar Virus [3].

The diagnosis is based on the criteria of Macdonald 2017 [4], which above all clinical, reinforced by radiological examinations including cerebral-medular MRI with the study of cerebrospinal fluid. Recently, it has been suggested that the levels of complement specific proteins such as $\mathrm{C} 1 \mathrm{q}$, C $3 d$, and C5b-9 in serum and CSF could potentially serve as new biomarkers to diagnose different MS subtypes and determine the activity of disease [5].

However, ocular involvement is sometimes the initiator of the disease, with optic neuritis which ranks first, followed by oculomotor palsies and internuclear ophthalmoplegia, nystagmus and uveitis being the least frequent [6].

\section{CONCLUSION}

Multiple sclerosis remains a mysterious pathology despite the progress of research, in particular its pathophysiology and its management, it is often disabling and the visual prognosis depends on the involvement of the optic nerve, hence the interest of an examination systematic ophthalmologic in front of any suspicion of multiple sclerosis.

\section{REFERENCES}

[1] Zara Shah, Mohammad Wasay, Burhan Z. Chaudhry, Sten Fredrikson. Multiple sclerosis in Pakistan: Current status and future. Perspective. Journal of the Neurological Sciences .27 July 2020

[2] Thomas RP Taylor et al. Prevalence and demographics of multiple sclerosis-associated uveitis: a UK biobank study. Multiple Sclerosis and Related Disorders 43 (2020) 102209.

[3] Confavreux C, Vukusic S. The clinical course of multiple sclerosis Handb Clin Neurol. 2014; 122: 343-369.

[4] R. Dobson et al. Multiple sclerosis - a review. European Journal of Neurology 2019, 26: 27-40.

[5] Maryam Gul, Amirhossein Azari Jafari et al, Molecular Biomarkers in Multiple Sclerosis and Its. Related Disorders: A Critical Review. Int. J. Mol. Sci. 2020, 21, 6020.

[6] Ling Chen 1, Lynn K Gordon et al. Ocular manifestations of multiple sclerosis Curr Opin Ophthalmol. 2005 Oct;16(5):315-20. 